PHYSICAL REVIEW E 71, 066405 (2005)

\title{
Role of the thermophoretic force on the transport of nanoparticles in dusty silane plasmas
}

\author{
Kathleen De Bleecker* and Annemie Bogaerts \\ PLASMANT, Department of Chemistry, University of Antwerp, Universiteitsplein 1, 2610 Wilrijk, Belgium \\ Wim Goedheer \\ FOM Institute for Plasma Physics “Rijnhuizen,” Association EURATOM-FOM, Trilateral Euregio Cluster, P.O. Box 1207, 3430 BE \\ Nieuwegein, The Netherlands \\ (Received 22 January 2005; published 21 June 2005)
}

\begin{abstract}
A comparison, based on numerical modeling, is made between the different forces experienced by nanoparticles present in a low pressure capacitively coupled parallel-plate silane $\left(\mathrm{SiH}_{4}\right)$ discharge. We investigate in particular the influence of the thermophoretic force on the spatial distribution of the nanoparticle density profiles, due to a thermal gradient in gas temperature induced by heating or cooling of the electrodes. A series of simulations with a one-dimensional fluid model are performed with asymmetrical variation of the electrode temperatures. It appears that the resulting density profile of the nanoparticles experiences a significant shift towards the cooler electrode as soon as a temperature difference is applied. Thus thermophoresis is capable of influencing the force balance of suspended nanoparticles in the plasma, even at relatively small electrode temperature differences.
\end{abstract}

DOI: 10.1103/PhysRevE.71.066405

PACS number(s): 52.27.Lw

\section{INTRODUCTION}

Dust particles, from a few nanometers up to several micrometers, have been observed in many processing plasmas used for etching, sputtering, or deposition of thin surface films. Until recently, the presence of dust was solely considered as a potential hazard, especially in the microelectronic and other surface processing technologies, as particles provide a significant source of film defects redeeming them as "killer" particles. Therefore early investigations mainly attempted at suppressing the particle growth or tried to avoid interference with the wafer surface. Currently, it seems that small particles can also have very interesting and useful properties, mainly due to their very small sizes (e.g., nanometer area), chemical composition, or uniform size distribution $[1,2]$. In the photovoltaic cell production, for example, the creation and inclusion of nanocrystalline silicon particles in the intrinsic layer of amorphous hydrogenated silicon ( $a$ $-\mathrm{Si}: \mathrm{H})$ can result in a significant increase of the product quality [3]. The newly formed material shows improved transport properties and stability against the light induced effect, also known as the Staebler-Wronski effect. In order to obtain a controlled growth and deposition of these particles on the substrate material, we obviously must understand the mechanisms behind their origin and their behavior, including a thorough understanding of their transport in the plasma. Comprehension of these aspects will help to advance the existent and future technological applications.

In this paper we use a modeling approach to analyze the interplay between the growth process of the dust grains in a radio-frequency silane $\left(\mathrm{SiH}_{4}\right)$ plasma and the different forces acting on the particles. Previously $[4,5]$ we developed a detailed chemical kinetics model describing the initial stage of

\footnotetext{
*Electronic address: kathleen.debleecker@ua.ac.be
}

particle generation, i.e., the nucleation, by incorporating silicon hydrides containing up to a maximum of 12 silicon atoms $\left(\mathrm{Si}_{n} \mathrm{H}_{m}\right.$ with $\left.n \leqslant 12\right)$. It appears that the negative ions are the most prominent species to consider if one wants to describe the earliest phase of dust formation, as the anions are electrostatically confined in the plasma center by the action of the ambipolar potential. Consequently, the anions have a longer residence time in the plasma compared to other plasma species, which enables them to trigger a whole reaction sequence that can ultimately lead to the formation of nanometer and micrometer sized particles. Here, particles in the nanometer area are of special interest. These particles are gradually grown from the nucleation species by directly coupling the growth kinetics model with the formation of nanoparticles of a predefined size. Due to the higher mobility of the electrons compared to positive ions, nanoparticles will quickly become negatively charged and can remain trapped in the plasma due to the action of the sheath electric fields which will repel negatively charged particles. In addition to the electrostatic force, nanoparticles can also experience other forces such as ion and neutral drag, gravity, and thermophoresis. Depending on the plasma conditions these forces can play an important role [6-9]. In a previous paper we have already analyzed the competition between the electrostatic and the ion drag force on submicrometer particles [10].

The focus of this paper is on the thermophoretic force arising from a thermal gradient in gas temperature by heating or cooling of one of the electrodes. In contrast to other transport studies [11,12], we use a one-dimensional (1D) fluid model originally developed by Akdim and Goedheer [13], which incorporates the influence of the dust on the discharge by accounting self-consistently for the coupling between the particle behavior and the changes they induce on the plasma properties. In order to make an estimate of the contribution of thermophoresis in a parallel-plate radio frequency silane discharge, the thermophoretic force is added in the transport 
equation of the dust. Although most industrial plasma applications are operated at moderate temperatures, some applications do require heating or cooling of the wafer. It seems that even in discharges with isothermal electrodes a thermal gradient can appear as a consequence of inhomogeneous gas heating by plasma power dissipation [14]. In that case a temperature gradient is created from the plasma center towards the plasma walls. Hence thermophoresis can be an important issue in many industrial applications. We note that thermophoresis can also be adopted as a physical method to remove the interfering charged dust particles from the processing area [2]. The particles are, for example, drawn to a liquid nitrogen cold finger, thereby using thermophoresis as a particle gettering technique [15]. In this paper we investigate the effect of the thermal gradient that arises from a temperature difference between two electrodes.

The analytical expressions of the different forces, the nanoparticle formation mechanisms, as well as a general overview of the 1D fluid model, are described in Sec. II. In Sec. III the calculated nanoparticle spatial distributions are discussed and the balance of the different forces on nanoparticles is analyzed. Finally, the conclusions are given in Sec. IV.

\section{MODEL DESCRIPTION}

In order to study the transport and thus the balance of the different forces acting on nanoparticles in a plasma and incorporate the growth kinetics of the dust species, as well as the large amount of other reactions occurring in reactive silane plasmas, a fluid modeling approach seems to be the best option. The fluid model is particularly useful to describe the large number of different species and chemical reactions that need to be considered in the nucleation part of the model. In the first paragraph a general overview of the applied fluid model is described. The following paragraphs deal with the description of the nanoparticle generation and the different components of the nanoparticle's flux. The last paragraph explains whether a dc bias should be considered that could arise from the nonuniform distribution of the negatively charged nanoparticles in the plasma.

\section{A. Fluid model}

The 1D self-consistent fluid model consists of particle balance equations for the different species (electrons, ions, radicals, molecules, and nanoparticles), an energy balance equation for the electrons, and the Poisson equation for the calculation of the electric field $E$ and the potential $V(E=$ $-\nabla V)$. The balance equations take all the different production and loss mechanisms of every species into account. The momentum conservation equation of small plasma species is replaced by a drift-diffusion approximation [16]. As a result, the transport equation for the neutral species (radicals and molecules) reduces to a diffusion term, while the particle flux of small charged species consists of two separate terms, a migration and a diffusive term. For the nanoparticles a separate transport equation is introduced, where other important forces, such as the thermophoretic force, are included (see
Sec. II C). We note that for the ions the drift-diffusion approximation can only be used when the applied electric rf field is replaced by an effective electric field, $E_{\text {eff }}$. By using this effective electric field the inertia effects of the ions are taken into account [16].

Further, an energy balance equation is incorporated for the electrons that includes ohmic heating and the loss of energy due to electron impact collisions. Besides the consideration of electron impact ionization, dissociation, excitation, and attachment reactions with molecules, the recombination of ions with electrons on the nanoparticle's surface is included by a recombination rate [13]. The electron energy distribution function (EEDF) is computed from the Boltzmann equation in the two-term approximation and is needed to obtain the reaction rate coefficients of every electronneutral collision, as well as the electron mobility and diffusion coefficients, as a function of the average electron energy, $\epsilon$. Other plasma species (ions, neutrals, and nanoparticles) are assumed to be in thermal equilibrium, hence no energy balance has to be considered. Finally, a sticking model describes the plasma-wall interaction of the neutral species.

For the solution of the particle balances, the electron energy balance, and the Poisson equation, the reactor is modeled by using a uniform mesh containing 128 grid points in the axial direction, i.e., the direction normal to the parallel plates, representing in this case an electrode spacing of $3 \mathrm{~cm}$. The numerical method used to solve the nonlinear coupled differential equations is based on an implicit finite-difference technique using the Scharfetter-Gummel exponential scheme [17]. More details concerning the algorithms and the discretization schemes can be found in [17]. The time step within an rf cycle $(50 \mathrm{MHz})$ is set to $2.5 \times 10^{-10} \mathrm{~s}$ (i.e., the rf cycle is divided in 80 time steps). In order to speed up the calculation, a longer time step of $10^{-5} \mathrm{~s}$ is adopted for the neutralneutral chemistry. Due to the large difference in time scale between the nanoparticle motion and the rf period, a different calculation cycle with its own specific time step is introduced for the computation of the nanoparticle transport and growth (see below, Sec. II C). An iterative procedure is implemented to couple both calculation cycles and ensures that the influence of the nanoparticle dynamics is taken into account in a self-consistent way. Convergence of the fluid model is finally reached when the relative change of the discharge parameters at the beginning of two succeeding rf cycles is less than $10^{-6}$.

The accuracy reached with the 1D fluid model will also depend upon the accuracy and the related uncertainty of the chemistry input data. The cross sections, needed for the calculation of the rate coefficients of inelastic electron collisions, typically have uncertainties in the range of $10-30 \%$, whereas the typical error of other reaction chemistry data seems to be of the order of $20-30 \%$ [16].

The chemistry of a "standard" silane plasma (i.e., without the inclusion of dust formation) has already been comprehensively compared to experiments at various combinations of process parameters and showed good agreement between modeling and experimental results [16].

\section{B. Nanoparticle formation mechanisms}

Dust generation in silane-based plasmas is generally assumed to proceed through a series of chemical reactions be- 
tween anions and silane molecules, better known as gas phase polymerization. The anions are believed to play a crucial role in the dust generation [18-20], as they are confined by the sheath electric fields, and hence have a longer residence time in the plasma. The typical residence times of other species, such as the neutrals, are not sufficient to trigger a whole reaction sequence. Time-resolved mass spectra also seem to validate the role of the anionic pathway in particle generation [21].

$\mathrm{SiH}_{3}{ }^{-}$and $\mathrm{SiH}_{2}{ }^{-}$are considered as the primary anions in our model, formed through electron induced dissociative attachment of silane:

$$
\mathrm{SiH}_{4}+e^{-} \rightarrow \mathrm{SiH}_{x}^{-}+\mathrm{H}_{4-x} \quad(x=2 \text { or } 3)
$$

and mainly leading to $\mathrm{SiH}_{3}{ }^{-}$formation. Both anions can in turn trigger successive chain reactions with (ground state or vibrationally excited) silane molecules, which polymerize into larger silyl anions $\mathrm{Si}_{n} \mathrm{H}_{2 n+1}^{-}$(starting from $\mathrm{SiH}_{3}{ }^{-}$), and silylene anions $\mathrm{Si}_{n} \mathrm{H}_{2 n}{ }^{-}$(starting from $\mathrm{SiH}_{2}{ }^{-}$):

$$
\begin{gathered}
\mathrm{Si}_{n} \mathrm{H}_{2 n+1}^{-}+\mathrm{SiH}_{4}^{(*)} \rightarrow \mathrm{Si}_{n+1} \mathrm{H}_{2 n+3}^{-}+\mathrm{H}_{2}, \\
\mathrm{Si}_{n} \mathrm{H}_{2 n}^{-}+\mathrm{SiH}_{4}^{(*)} \rightarrow \mathrm{Si}_{n+1} \mathrm{H}_{2 n+2}^{-}+\mathrm{H}_{2} .
\end{gathered}
$$

The extra internal energy of vibrationally excited $\mathrm{SiH}_{4}$ molecules is enough to overcome the endothermic nature of some of the chain reactions. The rate constant of the abovementioned polymerization reactions is expected to be in the order of $10^{-18} \mathrm{~m}^{3} \mathrm{~s}^{-1}$ [19], which is far below the suggested theoretical upper limit for ion-molecule reactions [22].

The detailed growth kinetics model cannot be extended up to an unlimited number of plasma species and is eventually stopped at species (ions and neutrals) containing 12 silicon atoms $\left(\mathrm{Si}_{12} \mathrm{H}_{m}^{x}\right)$, thereby incorporating a total of 69 different species in the model [4]. In analogy to our previous model [10], we have simulated the nanoparticle formation by introducing a step that immediately converts the largest negative species of the nucleation model $\left(\mathrm{Si}_{12} \mathrm{H}_{m}{ }^{-}\right)$into nanoparticles of a certain predefined size. The production rates of all anion reactions leading to species with more than 12 silicon atoms are used as a source term for the formation of nanoparticles of a specific given size. By incorporating the transport of submicrometer particles (described below), we are eventually able to determine where most nanoparticles of a certain size will reside in the plasma.

\section{Implementing transport of the dust particles}

Detailed knowledge of the different forces acting on nanoparticles is needed to determine the responses of the particles to varying external plasma parameters. In the next paragraphs the various forces that can have an effect on particle transport are summarized and in the last paragraph the resulting expression of the particle's flux is described.

\section{Gravity}

A particle in the plasma can be subject to gravity and the exerted force is proportional to the particle mass. For a spherical particle the gravitational force can be written as

$$
F_{g}=m_{d} g=\frac{4}{3} \pi r_{d}^{3} \rho_{d} g,
$$

where $m_{d}$ is the particle's mass, $\rho_{d}$ is the mass density of the particle, $r_{d}$ is the particle's radius, and $g$ the gravitational constant. For amorphous silicon $\rho_{d}$ is approximately 2.3 $\times 10^{3} \mathrm{~kg} / \mathrm{m}^{3}$ [14]. Gravity will pull the particles towards the lower electrode. However, for submicrometer particles gravity can be neglected, as the influence of other forces, such as the electrostatic force, are much stronger for particles smaller than a few micrometers.

\section{Electrostatic force}

The electrostatic force exerted by the electric field $E$ on a particle

$$
F_{e}=Q_{d} E
$$

pushes negatively charged particles from the electrodes towards the plasma center. The charge on the nanoparticle $Q_{d}$ can be determined by calculating the balance between the electron and ion current towards the nanoparticle's surface [10], and will strongly depend on particle size and plasma conditions. In a plasma, the electric field $E$ is much smaller in the plasma bulk compared to the electric field in the sheaths. The electrostatic force plays an important role, since it is responsible for the trapping of negatively charged particles in the radio-frequency discharge and is usually the most dominant force for small nanoparticles.

\section{Neutral drag}

The neutral drag force results from collisions with neutral gas molecules and causes a momentum transfer from the neutral gas to the nanoparticles. Using the kinetic gas theory, the neutral drag force can be approximated by [7]

$$
F_{n}=-\frac{4}{3} \pi r_{d}^{2} n_{n} m_{n} v_{t h}\left(v_{d}-v_{n}\right)
$$

where $n_{n}, m_{n}$, and $v_{t h}=\sqrt{8 k_{B} T / \pi m_{n}}$ are the neutral gas number density, the mass, and the average thermal velocity of the gas, respectively. Neutral drag will only be present when there exists a difference between the drift velocity of the particle, $v_{d}$, and the velocity of the gas, $v_{n}$. Since no convection is included in the present model $\left(v_{n}=0\right)$, the neutral drag force will simply act as a damping force on the velocity of the nanoparticles, and hence will result in a deceleration of the particles.

\section{Ion drag}

Similar to the momentum transfer from neutral gas molecules, positive ions driven by the electric field towards the plasma sheaths can transfer their momentum to the nanoparticles. This induced force is known as the ion drag force and consists of two components: the collection and the orbit force. The collection force represents the momentum transfer of all the ions that are collected by the particle. Each impinging ion transfers its original momentum, $m_{i} v_{i}$, making the collection force equal to [6], 


$$
F_{i}^{c}=\pi b_{c}^{2} n_{i} v_{s} m_{i} v_{i}
$$

where $v_{s}$ is the mean speed of the positive ions and $b_{c}$ the collection parameter.

The second component, the orbit force, is given by [6]

$$
F_{i}^{o}=4 \pi b_{\pi / 2}^{2} \Gamma n_{i} v_{s} m_{i} v_{i}
$$

where $b_{\pi / 2}$ is the impact parameter that corresponds to the deflection angle $\pi / 2$ and

$$
\Gamma=\frac{1}{2} \ln \left(\frac{\lambda_{L}^{2}+b_{\pi / 2}^{2}}{b_{c}^{2}+b_{\pi / 2}^{2}}\right)
$$

is the Coulomb logarithm. $\lambda_{L}$ represents the linearized Debye length [14] and is a combination of the electron and ion Debye lengths. The orbit force is caused by positive ions that do not reach the particle's surface, but transfer their momentum by Coulomb interactions.

\section{Thermophoresis}

Thermophoresis, consisting of the drift of particles in the presence of a gas temperature gradient in the discharge, can be induced by, e.g., heating or cooling of one of the electrodes and causes a particle movement to cooler regions of the plasma. Gas molecules in the hotter area have larger velocities and hence transfer more momentum to the nanoparticles than gas molecules in the cooler area of the plasma. This causes a net force in the direction of $-\nabla T_{\text {gas }}$. The thermophoretic force is given by [23]

$$
F_{t h}=-\frac{32}{15} \frac{r_{d}^{2}}{v_{t h}}\left(1+\frac{5 \pi}{32}(1-\alpha)\right) \kappa_{T} \nabla T_{\text {gas }},
$$

where $\nabla T_{\text {gas }}$ is the gas temperature gradient, and $\kappa_{T}$ is the translational part of the thermal conductivity. The thermal accommodation coefficient of the gas on the nanoparticle's surface, $\alpha$, mainly depends on the mass ratio of incident molecules and surface atoms, on the gas and surface temperatures, and on the adsorption energy [14]. A reasonable approximation for the accommodation coefficient is $\alpha \approx 1$ for surface and gas temperatures between 300 and $500 \mathrm{~K}$ [23].

\section{Resulting particle's flux}

The expression for the flux of the nanoparticles is obtained by assuming that the neutral drag force, which is considered here as a friction force, balances the sum of all other forces. Using the expression of the momentum loss frequency

$$
\nu_{m d}=\sqrt{2} \frac{p_{t o t}}{k_{B} T_{\text {gas }}} \pi r_{d}^{2} \sqrt{\frac{8 k_{B} T_{\text {gas }}}{\pi m_{d}}}
$$

and the mobility and diffusion coefficient

$$
\mu_{d}=\frac{Q_{d}}{m_{d} \nu_{m d}}
$$

$$
D_{d}=\mu_{d} \frac{k_{B} T_{g a s}}{Q_{d}},
$$

a kind of drift-diffusion expression for the flux of the nanoparticles is obtained [13],

$$
\begin{aligned}
\Gamma_{d}= & -\mu_{d} n_{d} E_{e f f}-D_{d} \frac{d n_{d}}{d x}-\frac{n_{d}}{\nu_{m d}} g \\
& +\sum \frac{n_{d} m_{i} v_{s}}{m_{d} \nu_{m d}}\left(4 \pi b_{\pi / 2}^{2} \Gamma+\pi b_{c}^{2}\right) \Gamma_{i} \\
& -\frac{32}{15} \frac{n_{d} r_{d}^{2}}{m_{d} \nu_{m d} v_{t h}} \kappa_{T} \nabla T_{\text {gas. }} .
\end{aligned}
$$

As a result, the nanoparticles can be treated with the same numerical procedures as the other charged species in the model.

As the drift velocity and the diffusion coefficient of the nanoparticles are much smaller than those of the positive and negative ions and the electrons, a different calculation cycle with a different time step is introduced for the computation of the density and the charge of the nanoparticles [13]. Hence first the transport equations of the ions, electrons, and the Poisson equation are solved during a number of rf cycles (i.e., the first calculation cycle), during which the nanoparticles do not move. During the second calculation cycle, the transport equation of the nanoparticles is solved with a greater time step, using the time-averaged electric field, and electron and positive ion fluxes. This causes, however, the creation of space charge regions, as the electron and positive ion densities do not change. These space charge regions will lead to instabilities in the solution of the Poisson equation and the electron transport equation when switching back to the first calculation cycle. Therefore the artificially generated space charge regions are corrected by adapting the positive ion density distributions prior to the next series of $\mathrm{rf}$ cycles, in which the nanoparticles are stationary and the ion and electron density profiles adapt themselves to the newly calculated density profile of the nanoparticles. By adopting this method a smaller computational effort is required.

\section{D. dc bias}

In a $1 \mathrm{D}$ model the time-dependent plasma characteristics will only vary along the axial direction. By not considering any radial dependency, the area of the parallel plates is assumed to be infinite. Hence usually no dc bias voltage has to be computed in a 1D model because a difference in size between the grounded and powered electrode, usually present in industrial reactors, cannot be considered. However, in this particular case, the nanoparticles will not be symmetrically distributed over the entire reactor, as an asymmetrical variation of the electrode temperatures causes the density profile to shift towards the colder electrode, i.e., in this case the powered electrode (see results, Sec. III). Since the nanoparticles are able to carry a large amount of negative charge, a dc current could be created if a difference between positive and negative fluxes is generated towards the powered electrode, where the majority of the nanoparticles are 
located close to the plasma-sheath boundary. As a result of this dc current flowing through the discharge, a bias voltage $V_{\mathrm{dc}}$ might arise at the powered electrode. To take this effect into account in our fluid model, the possibility of a dc bias voltage is added to the boundary conditions of the potential in the model. This dc bias voltage is calculated selfconsistently and adapted over the succeeding rf cycles until the fluxes of the ions and electrons, averaged over $1 \mathrm{rf}$ cycle, are of equal magnitude. Hence ultimately a potential will be created at the electrodes that causes the dc current to drop to zero. The voltage applied at the powered electrode is then described as

$$
V_{t}=V_{\mathrm{rf}} \sin \left(2 \pi \nu_{\mathrm{rf}} t\right)+V_{\mathrm{dc}}
$$

with $V_{\mathrm{rf}}$ the amplitude of the potential and $\nu_{\mathrm{rf}}$ the rf (set at $50 \mathrm{MHz}$ ). We note that the value of $V_{\mathrm{rf}}$ is adjusted until the dissipated power in the discharge matches the preset electrical power. The potential at the grounded electrode is set equal to zero.

In the present calculations we have investigated whether a difference between the fluxes of positive and negative charge is created at the cooled electrode, which would imply the extra incorporation of a dc bias voltage. Under the conditions applied in these simulations, we have computed that the nanoparticles are accumulating at a reasonable distance from the colder electrode, creating no remarkable difference in positive and negative flux calculated at the first grid point prior to the powered electrode. Hence no extra dc bias voltage is acquired, as it was computed to be less than $1 \mathrm{~V}$.

\section{RESULTS AND DISCUSSION}

In this section the results obtained with the 1D fluid model are presented. The simulated silane discharge is characterized by two parallel-plate electrodes separated by a gap of $3 \mathrm{~cm}$. The discharge is operated at a pressure of $40 \mathrm{~Pa}$, a power of $5 \mathrm{~W}$, a driving frequency of $50 \mathrm{MHz}$, and sustains an input gas flow of $20 \mathrm{sccm}$ pure silane. This specific set of discharge parameters is chosen in order to be able to model the regime in which experiments show that dust formation can play an important role [16]. To evaluate the influence of thermophoresis, the upper, grounded electrode temperature is varied between 300 and $500 \mathrm{~K}$, whereas the lower, powered electrode is kept at a constant temperature of $300 \mathrm{~K}$ in all calculations. We have chosen two specific cases of submicrometer particle sizes, i.e., $30 \mathrm{~nm}$ particles, which without the inducement of a thermophoretic force reside mostly in the center of the plasma, and $100 \mathrm{~nm}$ particles, which have a density profile that balances closer to the plasma boundaries [10].

\section{A. Density profiles of the nanoparticles}

In Fig. 1 the calculated normalized dust density profiles of $30 \mathrm{~nm}$ particles are shown at the operating conditions discussed above. In the figure, every plot represents a separate calculation for grounded electrode temperatures varied between 300 and $500 \mathrm{~K}$, while the rf electrode is held constant at $300 \mathrm{~K}$. Note that in the present calculations no steady state

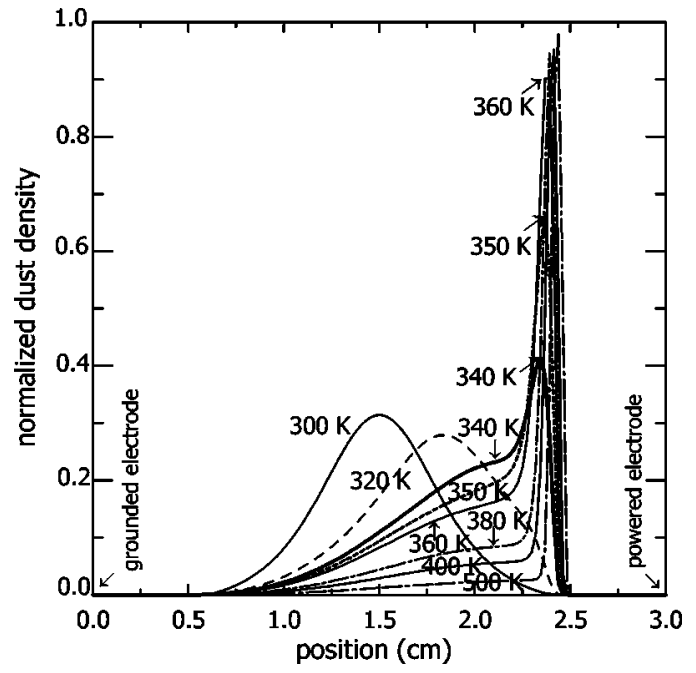

FIG. 1. Computed normalized spatial distribution of $30 \mathrm{~nm}$ particles for different temperature values of the grounded electrode (at $0 \mathrm{~cm}$ ) between 300 and $500 \mathrm{~K}$. The powered electrode (at $3 \mathrm{~cm}$ ) remains at the fixed temperature of $300 \mathrm{~K}$. In order to fit all the plots on the same figure, we have divided the 380,400 , and $500 \mathrm{~K}$ profiles by a factor of $1.5,2$, and 3 , respectively.

can be attained, as no loss processes are incorporated for the nanoparticles and hence they will continue to build up over time. Therefore we have adopted normalized density profiles in order to fit all the data on the same figure. In reality the nanoparticles will continue to grow due to coagulation and eventually gravity pulls the particles out of the discharge. From Fig. 1 we can clearly see that changing the grounded electrode temperature from 300 to $500 \mathrm{~K}$ has a significant effect on the density profile of the $30 \mathrm{~nm}$ particles, as it reacts to a shift of the balance of the different forces acting on the particles. When both electrodes remain at the fixed temperature of $300 \mathrm{~K}$, the majority of the $30 \mathrm{~nm}$ particles are confined in the bulk of the plasma and the plot exhibits a local maximum in the center of the discharge. As soon as a temperature difference is induced, the profile seems to gradually displace towards the colder electrode, thereby diminishing its concentration in the center between the two electrodes. This effect is already clearly visible at a temperature difference of $20 \mathrm{~K}$, where the maximum of the plot is slightly shifted towards the powered electrode. At a temperature difference of $40 \mathrm{~K}$, a peak is created in front of the rf electrode and this peak gradually increases at higher temperature differences, whereas the density in the bulk keeps decreasing.

A similar effect of electrode temperature difference on the calculated, normalized density profiles of $100 \mathrm{~nm}$ particles is illustrated in Fig. 2. An enlarged version of the centered rectangular area in Fig. 2(a) is depicted in Fig. 2(b) for a better visualization of the shift in density. When no thermophoresis is applied (i.e., both electrodes remain at $300 \mathrm{~K}$ ), most of the particles are segregated into two peaks of equal height, one near each plasma boundary. Hence two distinct layers of dust are visible that are symmetrically distributed over the reactor volume. If the grounded electrode is heated, while the $\mathrm{rf}$ electrode remains at a constant temperature, the $100 \mathrm{~nm}$ par- 

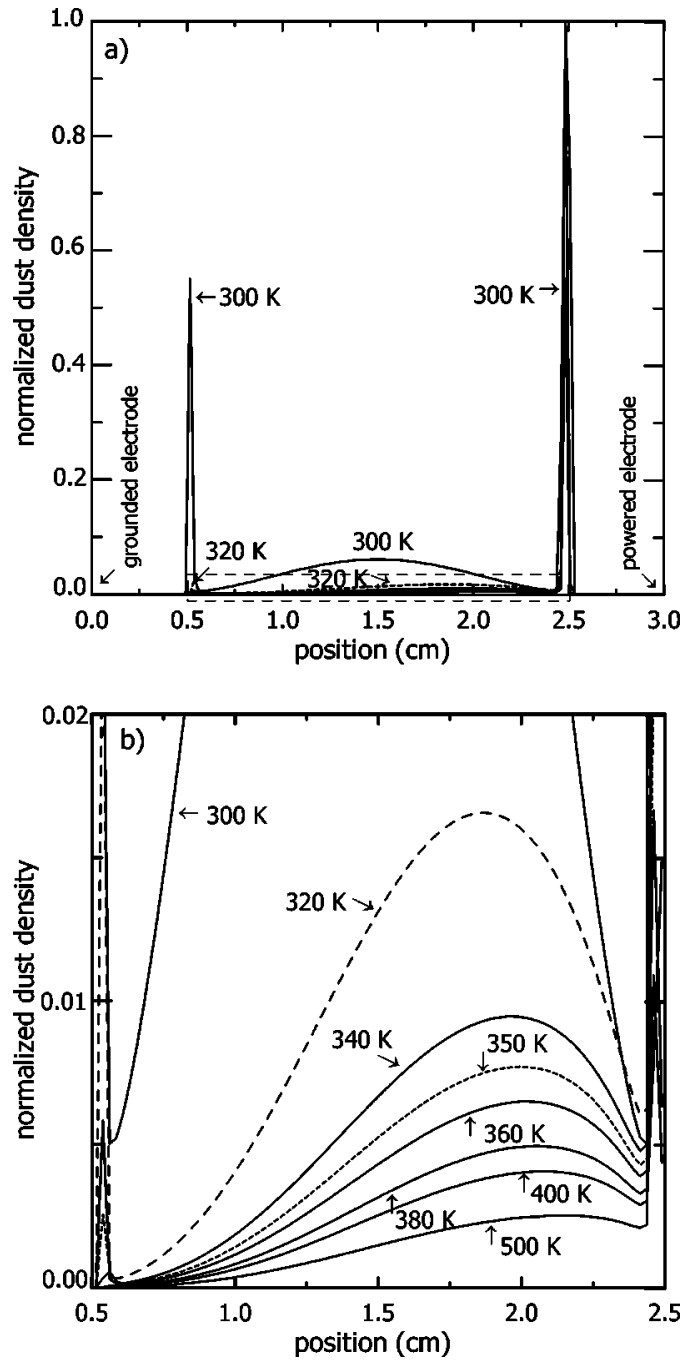

FIG. 2. Calculated normalized spatial distributions of $100 \mathrm{~nm}$ particles for different temperature values of the grounded electrode (at $0 \mathrm{~cm}$ ) between 300 and $500 \mathrm{~K}$. The powered electrode (at $3 \mathrm{~cm}$ ) is kept at a constant temperature of $300 \mathrm{~K}$. The plots have been divided by a factor in order to fit all the data on the same figure. In (b) we zoom in on the middle rectangular area, visualized by the dashed rectangle in plot (a).

ticles tend to move closer to the sheath boundary adjacent to the rf electrode. At a temperature difference of $20 \mathrm{~K}$, the peak near the powered electrode becomes considerably larger compared to the opposite peak close to the grounded electrode. Eventually at a temperature difference of $40 \mathrm{~K}$ and more, the peak adjacent to the grounded electrode disappears almost completely and a very pronounced peak close to the powered electrode is formed.

Hence our model predicts that even relatively small variations in electrode temperatures seem to have a strong influence on the spatial distribution profiles of particles in the submicrometer area. Due to the dynamic balance of the different forces acting on the nanoparticles, a dust particle will be suspended in some well-defined region of the plasma.

In order to validate these obtained results, we have compared our calculations with the experimental measurements obtained by Dorier et al. [25], who monitored and digitized the trapped silane dust particles for different grounded electrode temperatures with a charge-coupled device camera by illumination of the reactor volume with white light. There is very good agreement between the density profiles calculated with our fluid model and the ones obtained in the experiments. Indeed, the experiments reveal the same shift in density at the applied temperature differences and also lead to the depletion of the dust at the grounded electrode sheath. Other thermophoretic experiments by Jellum et al. [8], who have used aluminum, copper, or plasma generated carboncontaining dust particles of the order of $100-300 \mathrm{~nm}$ in diameter, also concur with the ones we obtain for $100 \mathrm{~nm}$ particles. By applying a temperature difference of $38 \mathrm{~K}$ to one of the two electrodes, almost all the dust particles accumulate at the cold electrode sheath. Furthermore, Bouchoule and Boufendi [24] have made experimental observations in an argon-silane plasma between two isothermal electrodes. By measuring the scattered laser intensity distribution between both electrodes, they were able to predict where the dust particles reside in the plasma as a function of time. Their observations also show a shift of the particle concentration towards the discharge sheaths and thus concur with the calculated $100 \mathrm{~nm}$ density profile that we obtain when no thermophoretic force is applied. Hence both the model and the experiments exactly predict the same tendencies.

In order to explain these specific calculated and observed density profiles and prove that the thermophoretic force is capable of influencing the spatial distribution of the dust particles to such a large extent, we require a calculation of the dynamic balance of the several forces acting on the particles as a function of the applied temperature gradients.

\section{B. The dynamic balance of the several forces acting on the nanoparticles}

In this section we will compare the magnitude of the thermophoretic force with the magnitude of other forces that govern the nanoparticles transport. For submicrometer particles no gravitational force has to be considered. The neutral drag force is assumed to only act as a damping force on the particulates and is thus in equilibrium with the sum of all other forces. Hence we suggest that the dynamic balance will be determined by the competition between the thermophoretic and two other important forces, i.e., the electrostatic and the ion drag force, and it determines where nanoparticles of a certain size will reside in the discharge. Barnes et al. [6] and Sommerer et al. [12] also propose that without any temperature gradients, the two most dominant forces acting on submicrometer particles are the electrostatic and the ion drag force.

\section{Forces acting on $30 \mathrm{~nm}$ particles}

In Fig. 3 the calculated electrostatic, ion drag, thermophoretic, and resulting net force on $30 \mathrm{~nm}$ particles are shown as a function of position in the plasma for varying grounded electrode temperatures. Note that the magnitude of the forces is very sensitive to the location within the plasma. In the sheath regions, where large electric fields are present, the electrostatic force will largely exceed the sum of all other 

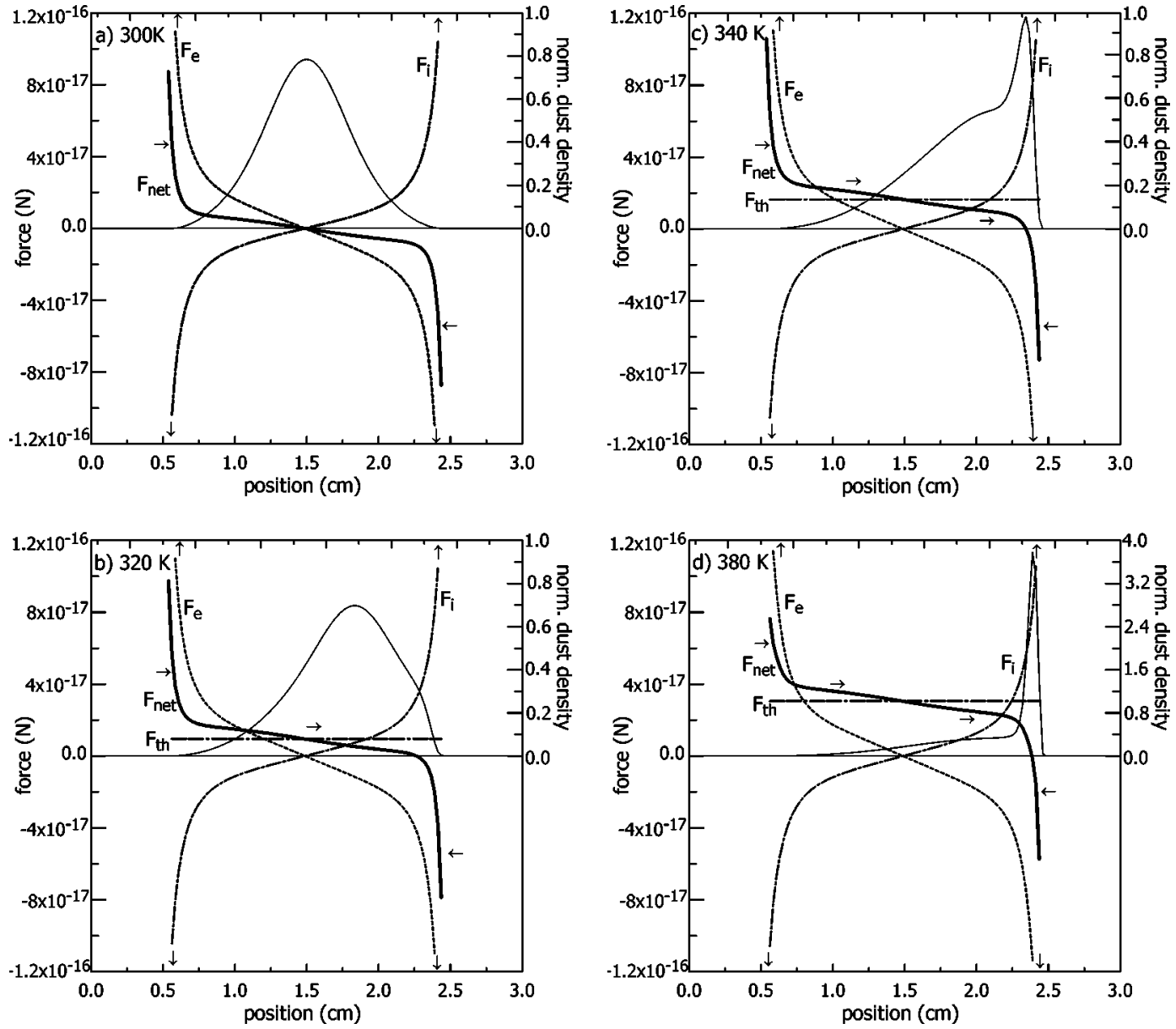

FIG. 3. Calculated electric force $\left(F_{e}\right)$, ion drag force $\left(F_{i}\right)$, thermophoretic force $\left(F_{t h}\right)$, and net force $\left(F_{\text {net }}\right)$ acting on $30 \mathrm{~nm}$ particles with a grounded electrode temperature (at $0 \mathrm{~cm}$ ) of (a) $300 \mathrm{~K}$; (b) $320 \mathrm{~K}$; (c) $340 \mathrm{~K}$; and (d) $380 \mathrm{~K}$. The powered electrode (at $3 \mathrm{~cm}$ ) is kept at a constant temperature of $300 \mathrm{~K}$. The forces are only shown in the bulk region of the discharge, i.e., between 0.5 and $2.5 \mathrm{~cm}$, where they attain the same order of magnitude. The direction of the net force, represented by a thicker solid line, is indicated by left and right arrows. The resulting density profile is also shown by a thin solid line, right axis.

forces and will repel the negatively charged particles from the walls and accelerate them towards the center of the plasma. However, the electric field $E$ is much smaller in the bulk of the plasma, hence under the present conditions the ion drag and thermophoretic force are likely to be of the same order of magnitude as the electrostatic force, and can in some cases even exceed the electrostatic force. The ion drag force will drive the nanoparticles symmetrically from the plasma centerline in the direction of the net positive ion flux, which is generally towards the sheath edges, while the thermophoretic force will push the particles towards the cooled electrode, i.e., in our case the powered electrode. In order to visualize this competition between the different forces in the plasma bulk, we have limited the plots in Fig. 3 to the central region of the plasma, i.e., between 0.5 and $2.5 \mathrm{~cm}$, respectively. The forces have been calculated for four different cases of grounded electrode temperature heating, namely the grounded electrode remains either at $300 \mathrm{~K}$ or is heated to, respectively, 320,340 , or $380 \mathrm{~K}$. The resulting spatial distribution of the $30 \mathrm{~nm}$ particles is also depicted in each plot by a thin solid line.

When no thermophoresis is applied [Fig. 3(a)], the electrostatic force $F_{e}$ is still large enough to maintain the par- ticles in the plasma bulk and the ion drag force does not seem to play a very important role in the transport of $30 \mathrm{~nm}$ particles. Therefore the particles mainly accumulate in the center of the discharge (see the additionally shown density profile).

When the grounded electrode is only slightly heated to a temperature of $320 \mathrm{~K}$ [Fig. 3(b)], the $30 \mathrm{~nm}$ particles immediately experience the extra applied thermophoretic force and the profile starts to slightly shift and bend towards the cooler electrode.

For even larger temperature gradients by heating of the grounded electrode to a temperature of 340 or $380 \mathrm{~K}$, respectively [Figs. 3(c) and 3(d)], the thermophoretic force really starts to dominate the transport of the $30 \mathrm{~nm}$ particles and drives them away from the heated electrode towards the cooled electrode to the position where the thermophoresis is balanced by the electrostatic force. At $340 \mathrm{~K}$ [Fig. 3(c)] the electrostatic force is still able to push some of the particles back towards the center of the discharge, but at $380 \mathrm{~K}$ [Fig. $3(d)]$ the thermophoretic force is the most dominant force at the centerline and moves the particles towards the plasmasheath boundary adjacent to the powered electrode, where a region of high electric fields is reached. At the point where 

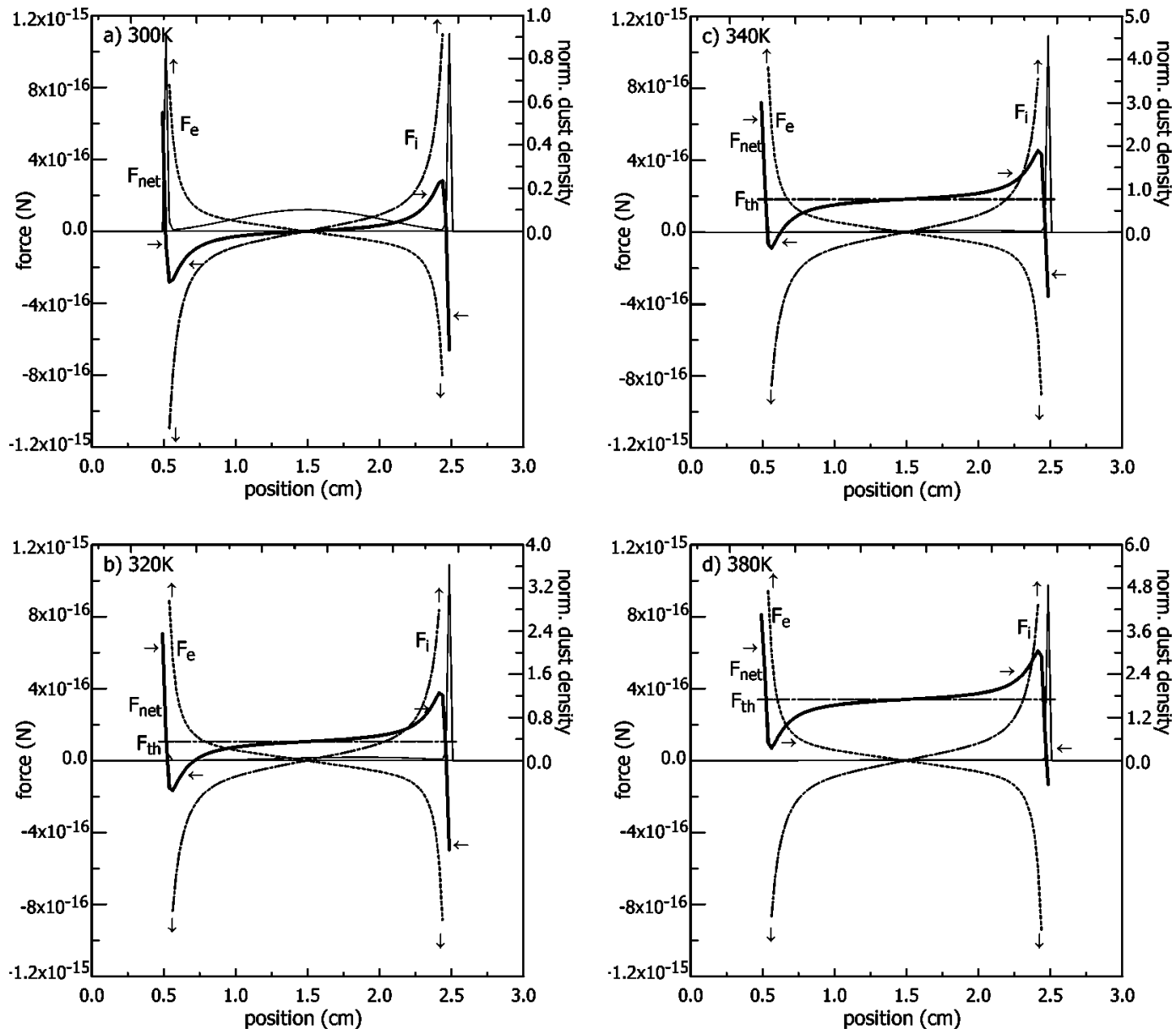

FIG. 4. Calculated electric force $\left(F_{e}\right)$, ion drag force $\left(F_{i}\right)$, thermophoretic force $\left(F_{t h}\right)$, and net force $\left(F_{n e t}\right)$ acting on $100 \mathrm{~nm}$ particles with a grounded electrode temperature of (a) $300 \mathrm{~K}$; (b) $320 \mathrm{~K}$; (c) $340 \mathrm{~K}$; and (d) $380 \mathrm{~K}$. The powered electrode (at $3 \mathrm{~cm}$ ) is kept at a constant temperature of $300 \mathrm{~K}$. The forces are only shown in the bulk region of the discharge, i.e., between 0.5 and $2.5 \mathrm{~cm}$. The direction of the net force, represented by a thicker solid line, is indicated by left and right arrows. The resulting density profile is also shown by a thin solid line, right axis.

the electrostatic, ion drag, and thermophoretic forces balance each other, i.e., where the net force goes through zero, a pronounced maximum is featured in the dust density profile.

\section{Forces acting on $100 \mathrm{~nm}$ particles}

Besides being sensitive to the location in the plasma, each of the forces also has a different power-law dependency on the nanoparticle radius (see above, Sec. II C) suggesting that the dominant force can change when the nanoparticles become larger. Figure 4 shows the calculated forces for $100 \mathrm{~nm}$ particles in the central region of the plasma at the same four specific cases of grounded electrode heating.

Without any gradients in gas temperature [Fig. 4(a)], it seems that the ion drag force plays a dominant role in the center of the plasma and drives the particles symmetrically from the plasma centerline towards the plasma boundaries until they begin to feel the "opposite" action of the electrostatic force. Most of the $100 \mathrm{~nm}$ particles will reside at the point where the electrostatic and ion drag force balance each other, visualized by two peaks of equal height close to the sheaths.
Similar to $30 \mathrm{~nm}$ particles, the thermophoretic force immediately starts to push the $100 \mathrm{~nm}$ particles away from the heated electrode, thereby diminishing the dust layer near the plasma-sheath of the grounded, heated electrode and heavily increasing the peak at the sheath region of the powered electrode in Figs. 4(b)-4(d). Hence the effect of the ion drag force in shifting the particles towards the heated electrode is completely canceled by the action of the thermophoretic force. In this case $F_{e}$ not only has to cancel the thermophoretic force $F_{t h}$, but in addition also the ion drag force $F_{i}$ at the sheath edge of the powered electrode. Hence the dust will be accumulated at the point where the three dominant forces balance each other, depicted by a pronounced, sharp peak near the plasma-sheath boundary adjacent to the powered electrode.

Hence we can conclude that it will be the dynamic competition between the different forces that causes a nanoparticle of a certain size to levitate in some well-defined region of the plasma. For $30 \mathrm{~nm}$ particles it seems that the competition between the thermophoretic and the electrostatic forces will mainly determine the location of the particles, whereas for $100 \mathrm{~nm}$ particles the ion drag force will also play an 
important role. As soon as a temperature difference is applied, the nanoparticles are mainly balanced by thermophoresis and the confining electrostatic force for $30 \mathrm{~nm}$ particles and by the thermophoretic, ion drag, and electrostatic force for $100 \mathrm{~nm}$ particles. Thus thermophoresis can strongly influence the spatial distribution of the particles in the plasma.

Finally, it should be mentioned that, according to the present study, thermophoresis can easily be used to drive the nanosized particles towards the cooler rf electrode, so away from the grounded electrode, by increasing the substrate temperature due to heating of the grounded electrode. Interference with the processing side can thereby be avoided. Hence thermophoresis can act as a particle suppression method and we can expect that it can be used as an efficient solution for the confinement of the contaminant dust particles as it prevents any contact with the wafer surface where, for example, the deposition process takes place. From the above results it seems that this method works well, even for small nanoparticles. The liquid nitrogen cold finger arrangement [15] is an experimental example of a particle scavenging technique that can even remove the disturbing particles from the discharge.

\section{SUMMARY AND CONCLUSIONS}

In this paper we have made a comparison between the different forces governing the transport of nanometer-sized particles that are electrostatically suspended in the plasma. The applied 1D self-consistent fluid model describes the nucleation chemistry, by including 69 different species, and couples it to the growth and transport of submicrometer particles. We have, in particular, studied the role of the thermophoretic force on the transport of two representative cases of nanoparticle sizes, i.e., 30 and $100 \mathrm{~nm}$. By varying the temperature of the grounded electrode between 300 and $500 \mathrm{~K}$, the density profile of 30 and $100 \mathrm{~nm}$ particles seems to gradually shift towards the cooler area at the plasma-sheath boundary adjacent to the powered electrode. Hence thermophoresis can be a very dominant force that can strongly influence the spatial distribution of the particles in the nanometer area, even at moderate temperature differences of $40 \mathrm{~K}$ or more. Furthermore, the calculated profiles concur with the experimental results obtained by Dorier et al. and Jellum et $a l$.

\section{ACKNOWLEDGMENTS}

K. De Bleecker is indebted to the Institute for the Promotion of Innovation through Science and Technology in Flanders (IWT-Vlaanderen) for financial support. W.G. receive support from the European Communities under the contract of Association between EURATOM and FOM, within the framework of the European Fusion Program and with financial support from NWO. Other financial support comes from the Belgian IAP program. The authors would like to thank Professor R. Gijbels for the interest he has shown in this work.
[1] A. Bouchoule, in Dusty Plasmas: Physics, Chemistry and Technological Impacts in Plasma Processing, edited by A. Bouchoule (Wiley, Chichester, UK, 1999).

[2] S. V. Vladimirov and K. Ostrikov, Phys. Rep. 393, 175 (2004).

[3] Y. Poissant, P. Chatterjee, and P. Roca i Cabarrocas, J. Appl. Phys. 94, 7305 (2003).

[4] K. De Bleecker, A. Bogaerts, R. Gijbels, and W. Goedheer, Phys. Rev. E 69, 056409 (2004).

[5] K. De Bleecker, A. Bogaerts, W. Goedheer, and R. Gijbels, IEEE Trans. Plasma Sci. 32, 691 (2004).

[6] M. S. Barnes, J. H. Keller, J. C. Forster, J. A. O'Neill, and D. K. Coultas, Phys. Rev. Lett. 68, 313 (1992).

[7] D. B. Graves, J. E. Daugherty, M. D. Kilgore, and R. K. Porteous, Plasma Sources Sci. Technol. 3, 433 (1994).

[8] G. M. Jellum, J. E. Daugherty, and D. B. Graves, J. Appl. Phys. 69, 6923 (1991).

[9] J. E. Daugherty, R. K. Porteous, and D. B. Graves, J. Appl. Phys. 73, 1617 (1993).

[10] K. De Bleecker, A. Bogaerts, and W. Goedheer, Phys. Rev. E 70, 056407 (2004).

[11] P. P. Rutkevych, K. Ostrikov, S. Xu, and S. V. Vladimirov, J. Appl. Phys. 96, 4421 (2004).

[12] T. J. Sommerer, M. S. Barnes, J. H. Keller, M. J. McCaughey, and M. J. Kushner, Appl. Phys. Lett. 59, 638 (1991).
[13] M. R. Akdim and W. J. Goedheer, J. Appl. Phys. 94, 104 (2003).

[14] J. Perrin, P. Molinàs-Mata, and Ph. Belenguer, J. Phys. D 27, 2499 (1994).

[15] H. Hahn and R. S. Averback, J. Appl. Phys. 67, 113 (1990).

[16] G. J. Nienhuis, W. J. Goedheer, E. A. G. Hamers, W. G. J. H. M. van Sark, and J. Bezemer, J. Appl. Phys. 82, 2060 (1997).

[17] J. D. P. Passchier and W. J. Goedheer, J. Appl. Phys. 73, 1073 (1993).

[18] L. Boufendi and A. Bouchoule, Plasma Sources Sci. Technol. 3, 262 (1994).

[19] J. Perrin, C. Böhm, R. Etemadi, and A. Lloret, Plasma Sources Sci. Technol. 3, 252 (1994).

[20] S. J. Choi and M. J. Kushner, J. Appl. Phys. 74, 853 (1993).

[21] Ch. Hollenstein, Plasma Phys. Controlled Fusion 42, R93 (2000).

[22] J. Perrin, O. Leroy, and M. C. Bordage, Contrib. Plasma Phys. 36, 3 (1996).

[23] L. Talbot, R. K. Cheng, R. W. Schefer, and D. R. Willis, J. Fluid Mech. 101, 737 (1980).

[24] A. Bouchoule and L. Boufendi, Plasma Sources Sci. Technol. 2, 204 (1993).

[25] J.-L. Dorier, Ch. Hollenstein, A. A. Howling, and U. Kroll, J. Vac. Sci. Technol. A 10, 1048 (1992). 